\title{
Systematic review and meta-analysis: the effect and safety of peritoneal dialysis in patients with end-stage diabetic kidney disease
}

\author{
Hao Wu ${ }^{1}$, Huiyou Zhou ${ }^{1}$, Qifan Zhang ${ }^{1}$, Ying Zhou ${ }^{1}$, Lizhen Fu ${ }^{1}$, Yijun Zhuang ${ }^{2}$ \\ ${ }^{1}$ Department of Internal Medicine of Donghu, The Second Affiliated Hospital of Hainan Medical University, Haikou, China; ${ }^{2}$ Department of \\ Nephrology, The Second Affiliated Hospital of Hainan Medical University, Haikou, China \\ Contributions: (I) Conception and design: H Wu, Y Zhuang; (II) Administrative support: H Zhou; (III) Provision of study materials or patients: H \\ Zhou, Q Zhang, Y Zhou, L Fu, Y Zhuang; (IV) Collection and assembly of data: H Wu, Q Zhang, Y Zhou, L Fu, Y Zhuang; (V) Data analysis and \\ interpretation: All authors; (VI) Manuscript writing: All authors; (VII) Final approval of manuscript: All authors. \\ Correspondence to: Yijun Zhuang. No. 48, Baishuitang Road, Longhua District, Haikou, China. Email: ZYJ66811325@163.com.
}

Background At present, peritoneal dialysis (PD) is widely used in the clinical treatment of patients with end-stage renal disease (ESRD), and comparison of the efficacy of PD and hemodialysis (HD) in the treatment of diabetic kidney disease (DKD) has been reported in a few clinical studies.

Methods: In this study, "dialysis", "peritoneal dialysis", "renal replacement therapy", "end-stage renal disease", "diabetic renal disease", and "efficacy and safety" were used as search terms in Chinese and English databases. According to RevMan 5.3 and Stata 13 software provided by the Cochrane Collaboration, a metaanalysis was performed.

Results: Four randomized controlled trials were included in this study, and 3 trials described the randomization method, 3 described allocation concealment in detail, and 2 used the blinding method. Compared with the HD treatment in the control group, the PD treatment in the experimental group can significantly reduce the hemoglobin of patients with end-stage DKD [Mean difference $(\mathrm{MD})=-0.13,95 \%$ confidence interval $(\mathrm{CI}):-0.21$ to $-0.04 ; \mathrm{P}=0.003<0.05]$ and Albumin level $(\mathrm{MD}=-0.10,95 \% \mathrm{CI}:-0.16$ to $-0.04 ; \mathrm{P}=0.002<0.05)$. Compared with the control group, the $\mathrm{PD}$ treatment in the experimental group significantly increased the serum creatinine and blood urea nitrogen levels in patients with end-stage DKD, but there was no significant difference in the effects of PD and HD treatment on serum creatinine levels (MD $=-0.30,95 \% \mathrm{CI}:-0.77$ to $0.16 ; \mathrm{P}=0.20>0.05),(\mathrm{MD}=1.93,95 \% \mathrm{CI}:-2.65$ to $6.51 ; \mathrm{P}=-0.41>0.05)$. In addition, $\mathrm{PD}$ treatment in the experimental group significantly increased the probability of malignant tumors in patients with end-stage DKD [odds ratio $(\mathrm{OR})=1.86,95 \% \mathrm{CI}: 1.64$ to $2.10 ; \mathrm{P}<0.00001$ ], and the difference was significant.

Discussions: This study used meta-analysis to confirm that PD can significantly improve the renal function of patients with end-stage DKD, but it can also increase the probability of protein loss and complications.

Keywords: Peritoneal dialysis (PD); hemodialysis (HD); end-stage diabetic kidney disease (DKD); safety; metaanalysis

Submitted Dec 11, 2021. Accepted for publication Feb 18, 2022.

doi: 10.21037/apm-22-50

View this article at: https://dx.doi.org/10.21037/apm-22-50 


\section{Introduction}

Diabetic kidney disease (DKD) is kidney disease caused by diabetes, with proteinuria as the main manifestation (1). With the aggravation of DKD, end-stage renal disease (ESRD) will gradually develop. The criteria for ESRD is a decrease in glomerular filtration rate to $15 \mathrm{~mL} / \mathrm{min} / 1.73 \mathrm{~m}^{2}$ or below, and when chronic kidney disease reaches stage 5 it becomes ESRD (2). There may be no significant discomfort in the early stages of ESRD, but with the progressive decline of renal function, toxins further accumulate in the body and can cause various symptoms of uremia, such as nausea, vomiting, anorexia, skin itching, ammonia odor, and edema, as well as a series of complications such as anemia $(3,4)$. At present, DKD is growing rapidly in developed countries, and the prevalence of DKD in China is also showing a rapid growth trend. From 2009 to 2012, the prevalence of DKD in patients with type 2 diabetes in China ranged from $30 \%$ to $50 \%$ in community patients and about $40 \%$ in hospitalized patients, and the corresponding prevalence of ESRD has also gradually increased (5).

Because ESRD can lead to severely impaired kidney function, a large amount of metabolic waste products cannot be normally excreted and deposited in the body, causing varying degrees of damage to various organs and tissues, which can cause organ failure and death. Therefore, promoting the effective excretion of toxic substances in ESRD patients and reducing the deposition of toxic substances are currently the mainstay of treatment and symptom alleviation for ESRD patients $(6,7)$. Treatments for diabetic ESRD include blood purification and kidney transplantation (8). hemodialysis (HD) can quickly and effectively remove small solutes and water from the patient's body. However, each dialysis requires the use of flaccid tube puncture, which has a great impact on the internal environment and hemodynamics of the body, resulting in faster loss of residual renal function, more opportunities for viral infection, and more frequent contact with medical staff (9). Due to factors such as renal origin, blood purification is currently the main method for the treatment of ESRD (10). Peritoneal dialysis (PD) uses the patient's own peritoneum to exchange solutes between blood and dialysate to remove excess fluid and metabolic waste from the body and maintain water, electrolyte, and acid-base balance (11). PD is less work-intensive and tends to preserve the patient's residual renal function (12). The advantages of PD are that compared with HD, PD has less effect on the cardiovascular system, has a better protective effect on residual renal function, is simple and easy to operate, can be used at home, results in more effective removal of middle molecular substances, has less impact on hemodynamics, and is suitable for elderly and young patients and patients with unstable cardiovascular conditions (13). However, it was found that PD has a higher incidence of peritonitis than HD and can lead to a large loss of nutrients such as protein, as well as increased weight and blood triglyceride levels in patients. Peritoneal fibrosis can be associated with post-dialysis, and patients are often in a state of volume overload (14). Furthermore, related complications of $\mathrm{PD}$ are more common in clinical practice, of which PD-related peritonitis (PDRP) is the most common and important complication after PD treatment in patients with end-stage DKD (15). The occurrence of PDRP will accelerate protein loss in patients with end-stage DKD, worsen renal function damage, and aggravate malnutrition, resulting in peritoneal sclerosis and a significantly reduced PD effect. PD has the advantages of good hemodynamic and cardiovascular stability and better protection of RRF in the treatment of diabetic ESRD patients (16). Some researchers used the Kaplan-Meier method to compare the survival rate and technical survival rate of diabetic and non-diabetic PD patients, and used Log rank to test for significance. The results showed that the 1-, 2-, 3- and 5-year survival rates of diabetic PD patients were lower than those of nondiabetic PD patients (Log rank 25.40, $\mathrm{P} \leq 0.001$ ); The survival rate of diabetic $\mathrm{PD}$ patients in patients aged $<65$ years was significantly lower than that of non-diabetic patients ( $\mathrm{Log}$ rank 13.36, $\mathrm{P}<0.001$ ); The technical survival rate of diabetic $\mathrm{PD}$ patients was similar to that of non-diabetic $\mathrm{PD}$ patients $(\mathrm{P}>0.05)$. It has been shown that the long-term survival rate of diabetic PD patients is lower than that of non-diabetic patients, but the technical survival rate is similar to that of non-diabetic patients, suggesting that PD is one of the suitable alternative treatment methods for diabetic ESRD patients (17). Dialysis patients with ESRD need to consider related factors such as the patient's condition and economic conditions when choosing a dialysis method. There is no absolute conclusion on which dialysis treatment method to choose in clinical practice.

With the widespread use of PD in the treatment of renalrelated diseases, PD has demonstrated overall efficacy and safety in patients with end-stage DKD. Therefore, in this study, the relevant literature related to PD for the treatment of patients with end-stage DKD were selected for metaanalysis, so as to evaluate the therapeutic effect and safety of PD for patients, aiming to provide some theory and data support for the application of PD in the clinical treatment 
of end-stage DKD. We present the following article in accordance with the PRISMA reporting checklist (available at https://apm.amegroups.com/article/view/10.21037/apm$22-50 / \mathrm{rc})$.

\section{Methods}

\section{Literature search}

The databases of China National Knowledge Infrastructure (CNKI) (1979-2021.4), Chinese Biomedical Literature (1994-2021.4), Cochrane Library (2005-2021.4), Medline (1948-2021.4), and Embase (1966.1-2021.4) were searched by computer. Published randomized controlled trials of PD in the treatment of DKD were searched, and the relevant studies in journals were manually searched. In this study, a compound logic retrieval method was used to select relevant documents. Chinese and English databases were search using the following terms: "dialysis", "peritoneal dialysis", "renal replacement therapy", "end-stage renal disease", "diabetic kidney disease", and "efficacy and safety". The search terms were freely combined. Using RevMan 5.3 and Stata 13 software provided by the Cochrane Collaboration, a meta-analysis was performed.

Preliminarily retrieved literature was screened firstly by reading the titles and abstracts, and non-conforming literature was excluded. A second screening was performed based on the inclusion and exclusion criteria, and a search engine was used to trace the included articles. Finally, the quality of the articles was evaluated by reading the full texts of the included studies and a third screening was performed. The search cutoff date for all articles was June 20, 2021.

\section{Literature inclusion and exclusion criteria}

The inclusion criteria were as follows: (I) published randomized controlled trials of HD and PD in the treatment of patients with end-stage DKD; (II) study subjects met the diagnostic criteria for end-stage DKD; (III) literature included patients' renal function indicators, nutritional level indicators, and the incidence of complications before and after treatment; (IV) literature had clear follow-up records.

The exclusion criteria were as follows: (I) conference speeches, review articles, study reports, lectures, and other literature; (II) studies in which the control group was not set or the control group was not treated with HD; (III) non-clinical randomized controlled trials; (IV) no followup records included; (V) full texts could not be obtained, studies with incomplete data, or repeated publications.

\section{Observation indicators}

The main therapeutic indexes in this study were serum creatinine, blood urea nitrogen, and other renal function indexes of patients before and after dialysis. The secondary efficacy indicators were nutritional level indicators such as hemoglobin and serum protein content. Indicators used to assess safety mainly included the incidence of various dialysis complications, such as peritoneal infection, cardiovascular and cerebrovascular disease, and hypoalbuminemia.

\section{Data extraction}

Data was extracted independently by 2 experts using Microsoft Excel. The 2 experts obtained consistent conclusions through discussion when they had inconsistent views. The data extracted for inclusion in the study were as follows: author of the study, research topic, research publication time, contact information of the author, sample size, basic characteristics of the research subjects, treatment intervention measures, treatment plan, evaluation index, efficacy, adverse reactions, etc. The quality of the literature was evaluated using the Cochrane Handbook for Systematic Reviews of Interventions 5.1.0.

\section{Risk of bias and quality assessment}

Two experts independently screened the literature based on the inclusion and exclusion criteria. The Cochrane Handbook for Systematic Reviews of Interventions 5.1.0 was used to evaluate the risk of bias in randomized controlled trials, including whether the random allocation method was used, whether allocation concealment was performed, whether the method was correct, whether blinding was used for the research subjects, treatment plan, and research results, selective reporting of results, and research data integrity. The above items were judged as "high risk bias", "low risk bias", and "unclear".

\section{Statistical methods}

Statistical analysis was performed using Stata SE 12.0 software (College Station, USA). The bias risk of the included studies was assessed using the risk of bias evaluation chart of RevMan 5.3 software. Mean difference (MD) was used for continuous variables, standardized mean 


\section{Identification of studies via databases and registers}

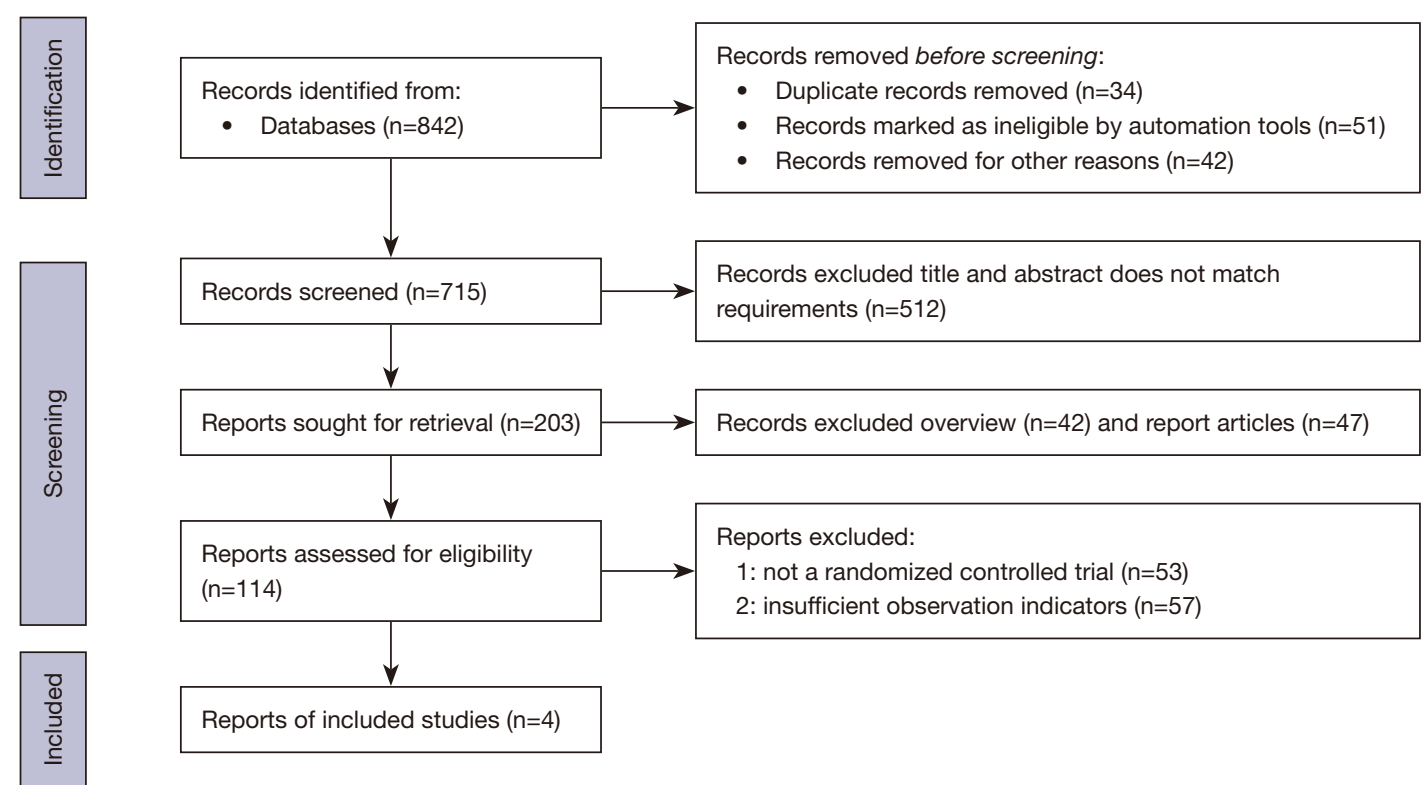

Figure 1 Literature retrieval flowchart.

difference (SMD) was used for discrete variables, and odds ratio (OR) was used for non-continuous variables. Each effect was expressed using a $95 \%$ confidence interval (CI). When $\mathrm{P}>0.01$ and $\mathrm{I}^{2}<50 \%$, a fixed-effect model was used for meta-analysis. When $\mathrm{P}<0.01$ and $\mathrm{I}^{2}>50 \%$, the randomeffect model was used for meta-analysis.

\section{Results}

\section{Search results and basic information of the included studies}

A total of 842 publications were obtained. Among them, 34 duplicate articles were excluded, 51 articles did not meet the automatic screening conditions, and 715 articles remained after 42 articles were deleted for other reasons. In addition, after reading the abstract and title, 512 articles were deleted, and then 114 articles were left after deleting reviews $(n=42)$ and research reports $(n=47)$. After reading the full text, 53 non-randomized controlled trials and 57 articles with insufficient observation indicators were excluded, and 4 studies were finally included in the metaanalysis (Figure 1).

Four studies met the inclusion criteria (18-21), involving 108,413 patients. In the 4 studies, the sample size ranged from 30 to 98,891 cases, and the age of the subjects was mostly concentrated between 45 and 65 years old. The sample size, experimental grouping, treatment plan, average age, and primary disease were described in detail in the 4 studies. The observation indexes in the studies included serum creatinine, blood urea nitrogen, hemoglobin, albumin level, and incidence of complications. Table 1 shows the basic characteristics of the included studies, and Figure 2 is the Jadad score distribution map of each randomized controlled trial included in the meta-analysis. The results showed that the Jadad scores were distributed between 2 and 4 points.

\section{Risk of bias evaluation of the included studies}

Figures 3,4 are the results of multiple risk bias assessments of the included studies drawn by RevMan 5.3 software. In this study, among the 4 randomized controlled trials, 3 (75\%) described the random allocation method and 3 (75\%) described allocation concealment in detail. There were 2 articles using the blinding method, accounting for $50 \%$, and no blinding method was used in the other studies.

\section{Meta-analysis of the effect of PD on bemoglobin}

Figure 5 shows a forest plot of the effect of different dialysis 
Table 1 Basic characteristics of the included studies

\begin{tabular}{|c|c|c|c|c|c|}
\hline Author & $\begin{array}{l}\text { Publication } \\
\text { year }\end{array}$ & Control group (HD) & Experimental group (PD) & $\begin{array}{l}\text { Average age } \\
\quad \text { (years) }\end{array}$ & Primary disease type \\
\hline Lee JH (19) & 2016 & 731 & 311 & $53.8 \pm 13.4$ & $\begin{array}{l}\text { Diabetes, hypertension, glomerular } \\
\text { inflammation, other }\end{array}$ \\
\hline Wang IK (21) & 2017 & 98,891 & 7340 & $61.2 \pm 14.4$ & $\begin{array}{l}\text { Diabetes, hypertension, glomerular } \\
\text { inflammation, other }\end{array}$ \\
\hline
\end{tabular}

$\mathrm{HD}$, hemodialysis; PD, peritoneal dialysis.

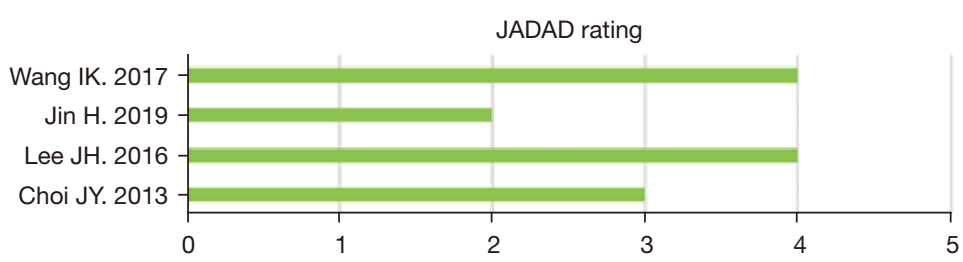

Figure 2 Jadad score distribution of the included studies.

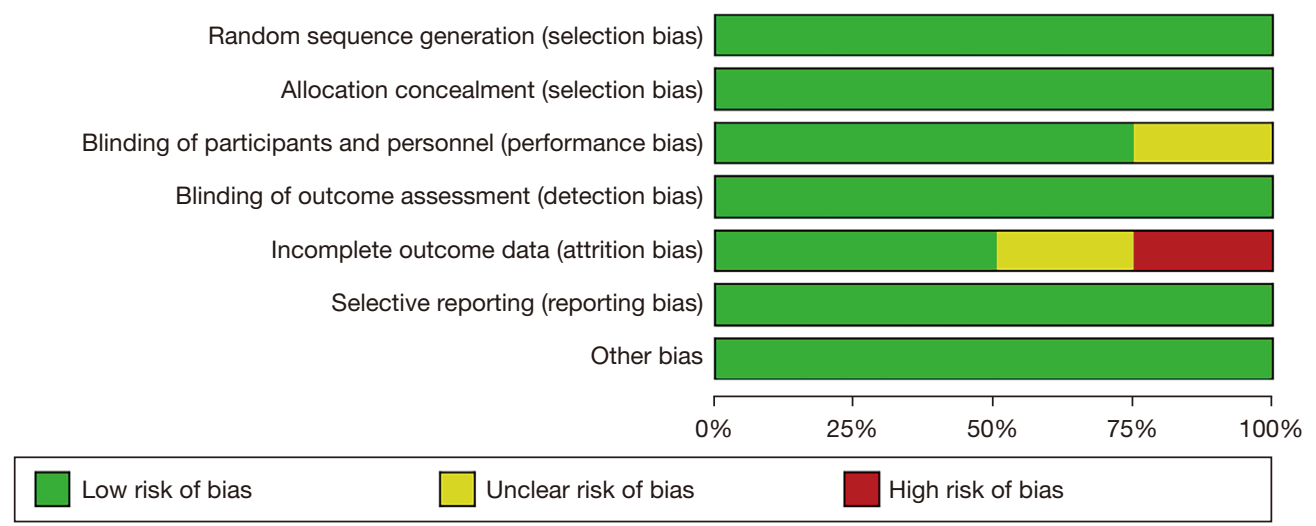

Figure 3 Results of the risk of bias assessment of the included studies.

methods on hemoglobin in the 2 groups of patients. Among the 4 studies included in the meta-analysis, 3 studies described the hemoglobin level after dialysis in the 2 groups in detail. The relevant data were extracted from the studies and heterogeneity analysis was performed for the hemoglobin level after treatment in patients with end-stage DKD. The results were $\mathrm{Chi}^{2}=3.25, \mathrm{df}=2, \mathrm{I}^{2}=38 \%<50 \%$; $\mathrm{P}=0.20$ for the hemoglobin level in the 2 groups, indicating that there was no heterogeneity between the hemoglobin levels of the subjects in the included studies. The fixed-effect model was used for statistical analysis, and it was found that PD treatment significantly reduced the hemoglobin level in patients with end-stage DKD compared with the control group ( $M D=-0.13,95 \%$ CI: -0.21 to $-0.04 ; \mathrm{P}=0.003<0.05$ ).

\section{Meta-analysis of the effect of PD on albumin}

Figure 6 shows a forest plot of the effect of PD on albumin 


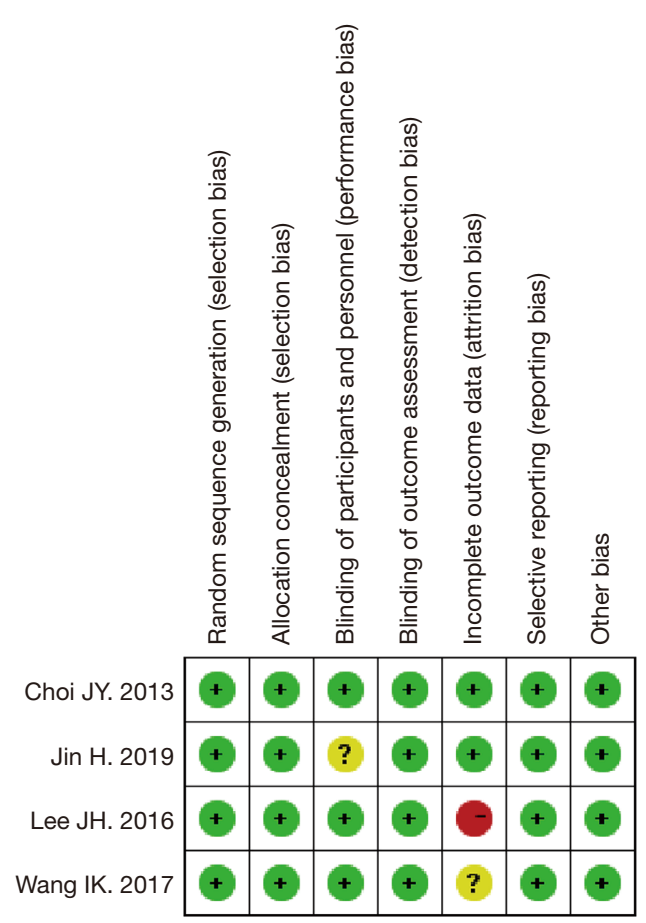

Figure 4 Results of multiple risk bias assessments of the included studies. + represents low risk bias; - represents high risk bias; ? represents unclear risk bias. in both groups. Among the 4 studies included in this meta-analysis, 3 described the albumin level after dialysis treatment in detail in the 2 groups. The relevant data of these 3 studies were extracted and heterogeneity analysis was performed for the albumin level after treatment in patients with end-stage DKD. The results were $\mathrm{Chi}^{2}=0.45$, $\mathrm{df}=2, \mathrm{I}^{2}=0 \%<50 \% ; \mathrm{P}=0.80$ for the albumin level in the 2 groups, indicating that there was no heterogeneity in the albumin level among the included studies. The fixed-effect model was used for statistical analysis, and it was found that PD treatment significantly reduced the albumin level in patients with end-stage DKD compared with the control group ( $\mathrm{MD}=-0.10,95 \% \mathrm{CI}:-0.16$ to $-0.04 ; \mathrm{P}=0.002<0.05)$.

\section{Meta-analysis of the effect of PD on serum creatinine}

Figure 7 shows a forest plot of the effect of PD on serum creatinine in both groups. Among the 4 studies included in this meta-analysis, 2 described the serum creatinine level after dialysis in detail in the 2 groups. The relevant data of these 2 studies were extracted and heterogeneity analysis was performed on the serum creatinine level after treatment in patients with end-stage DKD. The results were $\mathrm{Chi}^{2}=0.03$,

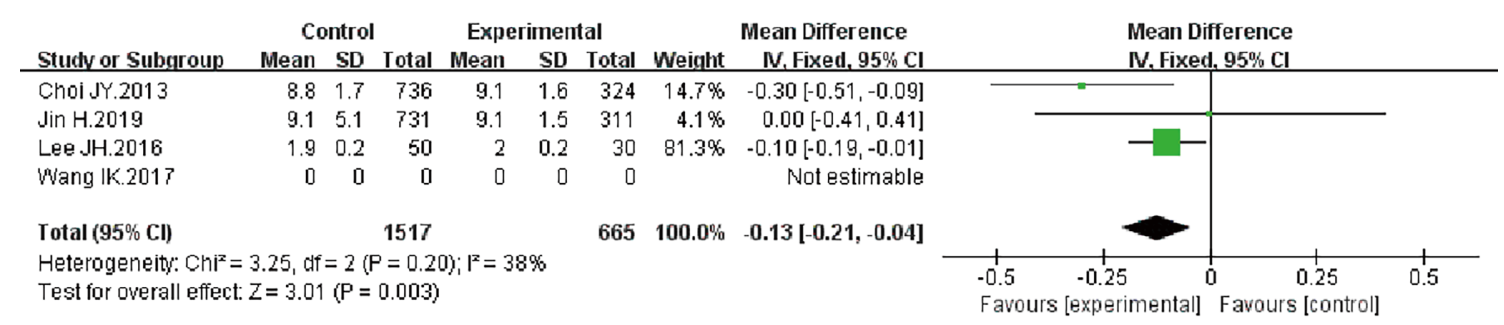

Figure $\mathbf{5}$ Forest plot of hemoglobin levels after dialysis treatment in patients.

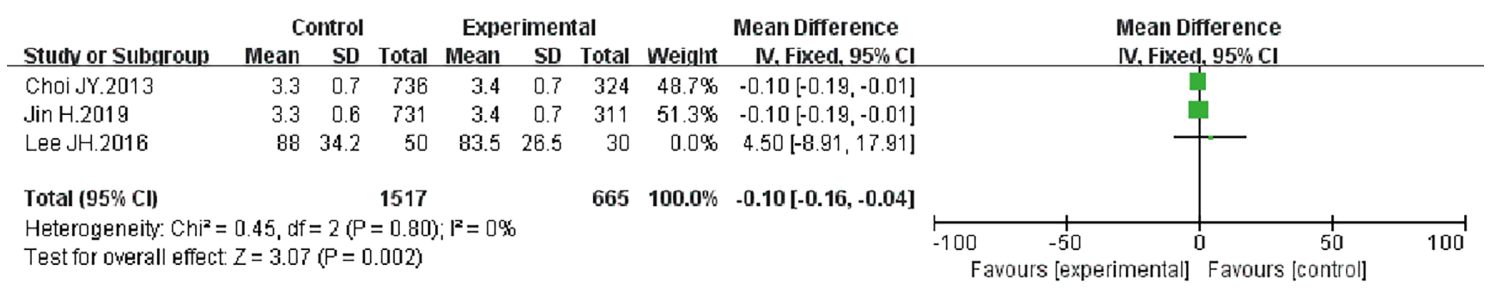

Figure 6 Forest plot of the effect of dialysis treatment on albumin in patients. 


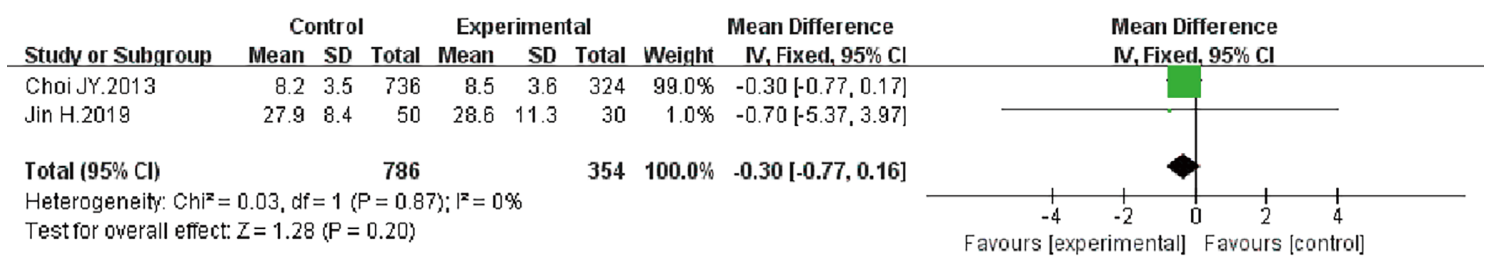

Figure 7 Forest plot of the effect of dialysis treatment on serum creatinine in patients.

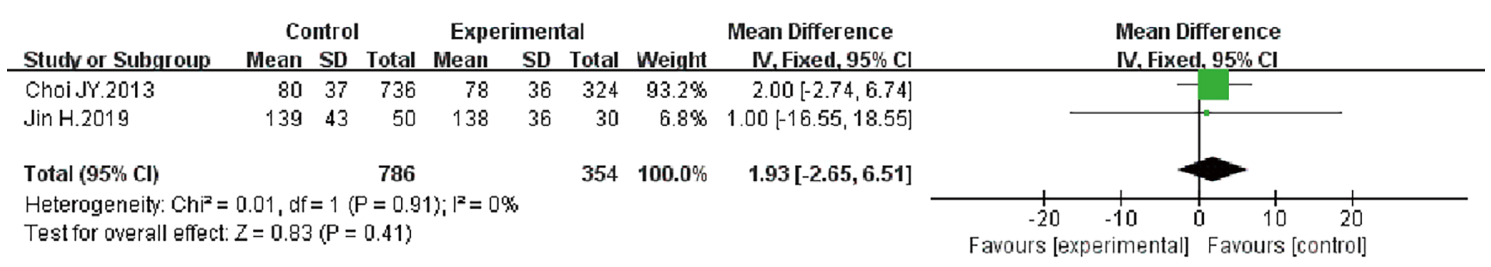

Figure 8 Forest plot of the effect of dialysis treatment on blood urea nitrogen in patients.

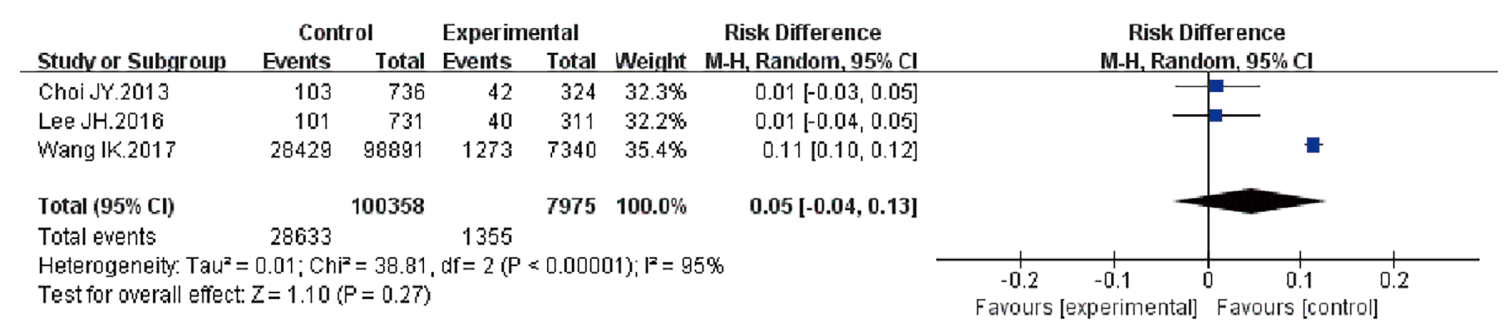

Figure 9 Forest plot of different dialysis treatments on the incidence of congestive heart failure in patients.

$\mathrm{df}=1, \mathrm{I}^{2}=0 \%<50 \% ; \mathrm{P}=0.87$ for the serum creatinine level in the 2 groups, indicating that there was no heterogeneity in the serum creatinine level in the included studies. The fixed-effect model was used for statistical analysis, and it was found that there was no significant difference in the serum creatinine level between the 2 groups of patients with endstage DKD after PD treatment and HD treatment (MD $=-0.30,95 \%$ CI: -0.77 to $0.16 ; \mathrm{P}=0.20>0.05)$.

\section{Meta-analysis of the effect of PD on blood urea nitrogen}

Figure 8 shows a forest plot of the effect of PD on blood urea nitrogen in both groups. Among the 4 studies included in this meta-analysis, 2 described the blood urea nitrogen level after PD in detail in the 2 groups. The relevant data of these 2 studies were extracted and heterogeneity analysis was performed on the blood urea nitrogen level of patients with end-stage DKD at each time point of treatment. The results showed that after treatment with different dialysis methods, $\mathrm{Chi}^{2}=0.01, \mathrm{df}=1, \mathrm{I}^{2}=0 \%<50 \% ; \mathrm{P}=0.91$ for blood urea nitrogen level in the 2 groups, indicating that there was no heterogeneity in the blood urea nitrogen level among the included studies. The fixed-effect model was used for statistical analysis, and it was found that there was no significant difference in the blood urea nitrogen level between the experimental group and the control group after dialysis treatment in patients with end-stage DKD ( $M D=1.93,95 \%$ CI: -2.65 to $6.51 ; \mathrm{P}=0.41>0.05)$.

\section{Meta-analysis of the effect of PD on the incidence of complications in patients}

Forest plots showing the effect of PD on the incidence of complications in the 2 groups are illustrated in Figures 9-12. Among them, Figure 9 is the forest plot of the effect of different dialysis treatments on the incidence of congestive heart failure. Figure 10 is the forest plot of the effect of different dialysis treatments on the incidence of malignant 


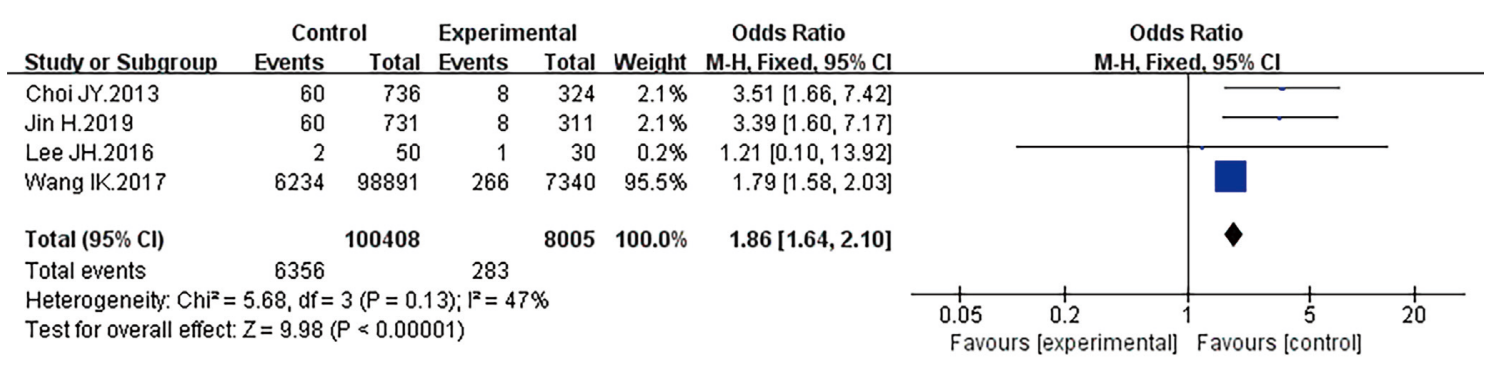

Figure 10 Forest plot of different dialysis treatments on the incidence of malignant tumors in patients.

\begin{tabular}{|c|c|c|c|c|c|c|c|c|c|}
\hline Study or Subgroup & $\begin{array}{l}\text { Contr } \\
\text { Events }\end{array}$ & $\begin{array}{l}\text { ol } \\
\text { Total }\end{array}$ & $\begin{array}{l}\text { Experim } \\
\text { Events }\end{array}$ & $\begin{array}{l}\text { ntal } \\
\text { Total }\end{array}$ & Weight & $\begin{array}{c}\text { Odds Ratio } \\
\text { M-H. Random. } 95 \% \mathrm{Cl}\end{array}$ & \multicolumn{3}{|c|}{$\begin{array}{c}\text { Odds Ratio } \\
\text { M-H, Random, } 95 \% \mathrm{Cl}\end{array}$} \\
\hline Choi JY.2013 & 88 & 736 & 30 & 324 & $46.8 \%$ & $1.33[0.86,2.06]$ & & & \\
\hline Jin H.2019 & 88 & 731 & 30 & 311 & 46.79 & $1.28[0.83,1.99]$ & & & \\
\hline Lee JH.2016 & 1 & 50 & 5 & 30 & $6.5 \%$ & $0.10[0.01,0.92]$ & & & \\
\hline Total $(95 \% \mathrm{Cl})$ & \multicolumn{3}{|c|}{1517} & 665 & $100.0 \%$ & $1.11[0.61,2.00]$ & & & \\
\hline Total events & \multicolumn{4}{|c|}{$177 \quad 65$} & & & & & \\
\hline \multicolumn{7}{|c|}{$\begin{array}{l}\text { Heterageneity: } \mathrm{Tau}^{2}=0.15 ; \mathrm{Chi}^{2}=5.09, \mathrm{df}=2(\mathrm{P}=0.08) ; \mathrm{I}^{2}=61 \% \\
\text { Test for owerall effect: } Z=0.34(\mathrm{P}=0.74)\end{array}$} & 0.002 & $\begin{array}{ccc}0.1 & 1 & 10 \\
\text { perimental] } & \text { Favours [co }\end{array}$ & 500 \\
\hline
\end{tabular}

Figure 11 Forest plot of the incidence of cerebrovascular disease in patients with different dialysis treatments.

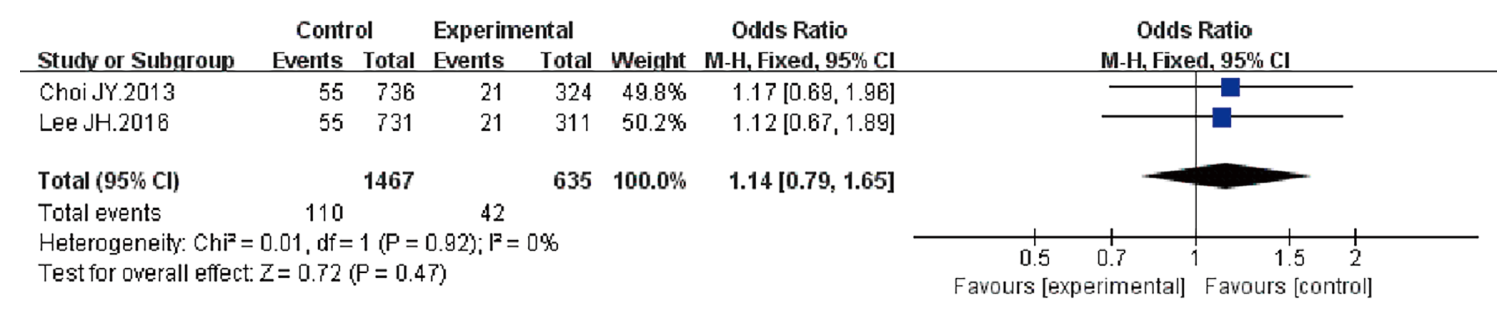

Figure 12 Forest plot of different dialysis treatments on the incidence of peptic ulcer in patients.

tumors. Figure 11 is the forest plot of the effect of different dialysis treatments on the incidence of cerebrovascular diseases. Figure 12 is the forest plot of the effect of different dialysis treatments on peptic ulcer. Among the 4 studies included in this meta-analysis, 3 studies described the occurrence of congestive heart failure after dialysis treatment in the 2 groups in detail, 4 studies described the occurrence of malignant tumor after dialysis treatment in the 2 groups in detail, 3 studies described the occurrence of cerebrovascular disease after dialysis treatment in the 2 groups in detail, and 2 studies described the occurrence of peptic ulcer disease after dialysis treatment in the 2 groups in detail. The corresponding data of the relevant studies were extracted to analyze the heterogeneity of the incidence rate of various complications after treatment in patients with end-stage DKD. The results of Figure 9 showed that the incidence rate of congestive heart failure in the 2 groups after different dialysis treatments was $\mathrm{Chi}^{2}=38.81, \mathrm{df}=2, \mathrm{I}^{2}=95 \%>50 \% ; \mathrm{P}<0.00001$, indicating that among the 3 studies involving dialysis treatment for endstage DKD, there was significant heterogeneity between the data of the 2 groups. Statistical analysis was performed using the random-effect model, and it was found that there was no significant difference in the probability of congestive heart failure after different dialysis treatments between the experimental group and the control group $(\mathrm{OR}=0.05,95 \%$ CI: -0.04 to $0.13 ; \mathrm{P}=0.27>0.05$ ).

The results of Figure 10 showed that the incidence rate of malignant tumor in the 2 groups after different dialysis treatments was $\mathrm{Chi}^{2}=5.68, \mathrm{df}=3, \mathrm{I}^{2}=47 \%<50 \% ; \mathrm{P}=0.13$, indicating that among the 4 studies involving the dialysis treatment of end-stage $\mathrm{DKD}$, there was no significant 


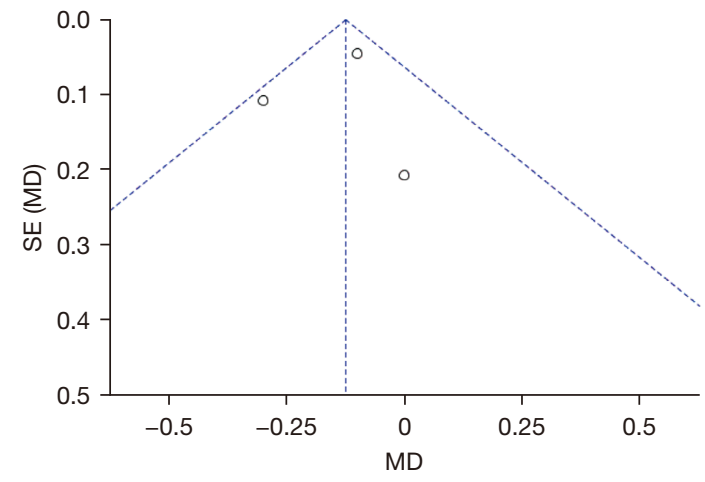

Figure 13 Funnel plot of hemoglobin level. MD, mean difference; SE, standard error.

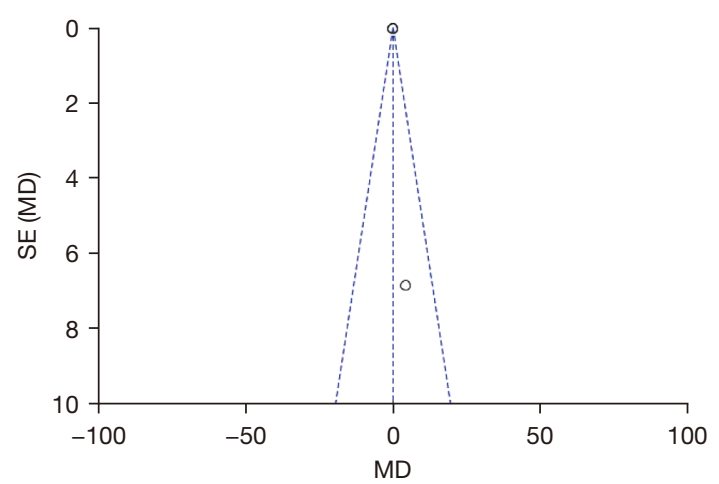

Figure 14 Funnel plot of albumin level. MD, mean difference; SE, standard error.

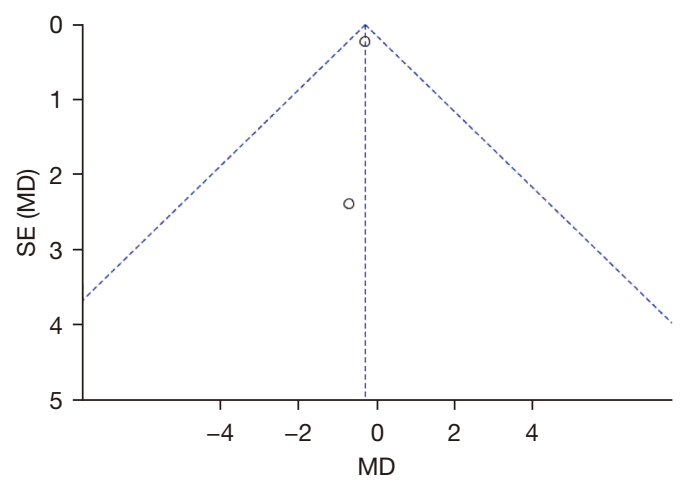

Figure 15 Funnel plot of serum creatinine level. MD, mean difference; SE, standard error.

heterogeneity between the data of the 2 groups. The fixedeffect model was used for statistical analysis. It was found that compared with the control group, the probability of malignant tumor after PD treatment in the experimental group was significantly increased, and the difference between the 2 groups was significant ( $\mathrm{OR}=1.86,95 \% \mathrm{CI}$ : 1.64 to $2.10 ; \mathrm{P}<0.00001)$.

The results of Figure 11 showed that the incidence rate of cerebrovascular disease in the 2 groups after different dialysis treatments was $\mathrm{Chi}^{2}=5.09, \mathrm{df}=2, \mathrm{I}^{2}=61 \%>50 \%$; $\mathrm{P}=0.08$, indicating that there was significant heterogeneity among the 3 studies involving cerebrovascular disease after dialysis treatment in end-stage DKD patients. Statistical analysis was performed using the random-effect model. It was found that there was no significant difference in the probability of cerebrovascular disease between the experimental group and the control group after different dialysis treatments $(\mathrm{OR}=1.11,95 \% \mathrm{CI}: 0.61$ to 2.00; $\mathrm{P}=0.74>0.05$ ).

The results of Figure 12 showed that the incidence rate of peptic ulcer in the 2 groups after different dialysis treatments was $\mathrm{Chi}^{2}=0.01, \mathrm{df}=1, \mathrm{I}^{2}=0 \%<50 \% ; \mathrm{P}=0.92$, indicating that there was no significant heterogeneity in the 2 studies involving peptic ulcer after dialysis treatment in end-stage DKD patients. The fixed-effect model was used for statistical analysis, and it was found that there was no significant difference in the probability of peptic ulcer after different dialysis methods between the experimental group and the control group (OR $=1.14,95 \% \mathrm{CI}: 0.79$ to 1.65 ; $\mathrm{P}=0.47>0.05)$.

\section{Publication bias results}

Figures 13-17 are funnel plots of publication bias of the included studies. Figures 13-17 are the funnel plots generated according to the information of serum creatinine, blood urea nitrogen, hemoglobin, albumin level, and the incidence rate of complications of the included studies. The circles of some included studies were basically concentrated on the midline and were generally symmetrical with the midline, indicating that there was no bias in the included publications, and the conclusions were relatively reliable.

\section{Discussion}

Patients with end-stage DKD often have severe complications and require renal replacement therapy. $\mathrm{HD}$ and $\mathrm{PD}$, as the most commonly used renal replacement therapy methods, can both replace part of the kidney function and improve the prognosis of patients, but they have different effects on patients with end-stage diabetic nephropathy during 


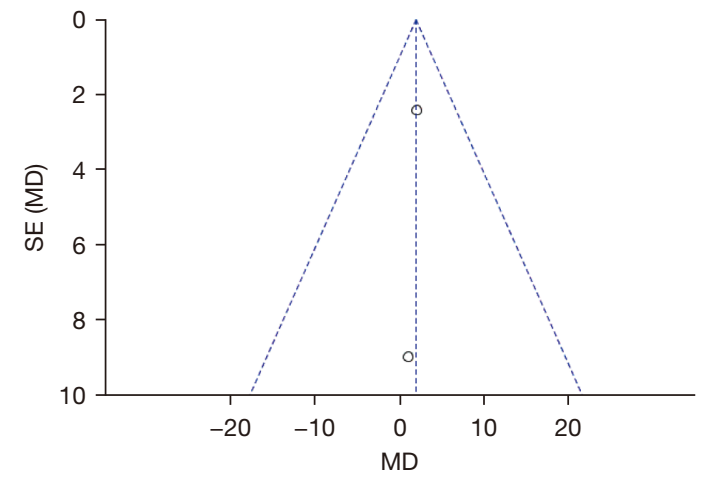

Figure 16 Funnel plot of blood urea nitrogen level. MD, mean difference; SE, standard error.

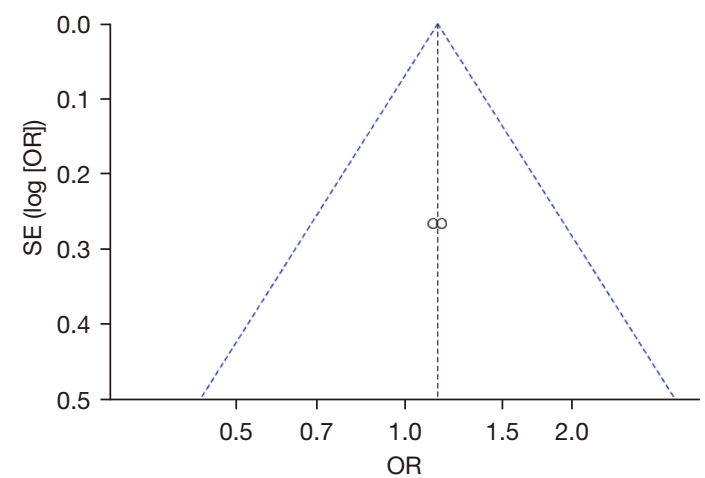

Figure 17 Funnel plot of the incidence of complications. MD, mean difference; SE, standard error.

the treatment process (22). At present, there is no absolute conclusion on which dialysis method is most suitable for patients with end-stage DKD. A total of 4 articles were included in this systematic review and meta-analysis to evaluate the therapeutic effect and safety of PD in patients with end-stage DKD. There were 3 articles that described the randomization method, 3 articles that described allocation concealment in detail, and 2 articles that used the blinding method. Biased results may be due to unclear randomization methods of the study and subjectivity bias of the physicians $(23,24)$.

The results of this study showed that PD significantly increased serum creatinine and blood urea nitrogen levels in patients with end-stage DKD, but there was no significant difference in the effect of HD on serum creatinine and blood urea nitrogen levels. The results were different from the conclusions of some related studies, which may be related to the small number of studies included in this meta- analysis. The PD treatment method has the characteristics of persistence and is very close to the operation mode of the human body, so it has the advantage of stabilizing blood circulation. Moreover, the PD treatment is relatively simple and has a protective effect on residual renal function (25). The study by Ranganathan et al. (2017) (26) demonstrated that the renal protective effect of PD in patients with end-stage DKD is because PD uses the patient's own peritoneum as the dialysis membrane, and through the principles of diffusion, convection, and ultrafiltration, the dialysate is periodically injected into the peritoneal cavity and the dialysate waste is discharged, so as to achieve the effect of metabolic exchange. This shows that compared with HD treatment, PD treatment can better protect the residual renal function of patients with end-stage DKD, to achieve a better removal of excess water and metabolites in the body. Xue et al. (2019) (27) used a meta-analysis method to analyze the mortality risk of PD and HD in patients with end-stage DKD. The results found that the mortality rate of $\mathrm{PD}$ in patients with end-stage DKD was higher than that of $\mathrm{HD}$, and the mortality risk of PD in patients in Asian countries was significantly increased, indicating that the use of HD can reduce the mortality of end-stage DKD patients. This is different from the results of this study. The reason for the analysis may be that this study only analyzed the death risk of patients with different treatments, while this study analyzed the biochemical indicators and complications of patients after two treatments. The research focus and purpose of the two literatures are different, resulting in some differences in their research conclusions.

In addition, PD was shown to significantly reduce hemoglobin and albumin levels in patients with end-stage DKD after 1 week, 1 month, and 3 months of treatment, thus indicating that PD may cause inflammatory-related protein loss and long-term chronic inflammation in patients (28). It was pointed out that patients with end-stage diabetes are prone to protein-energy malnutrition (PEM), microinflammatory state, and infection (29). Albumin is an important indicator to reflect the nutritional status of the human body. When albumin is reduced, it can lead to low immunity of the body, and it is easy to be accompanied by infection. Albumin is also an important substance to stabilize plasma colloid osmotic pressure. Long-term low albumin can cause edema and serous cavity effusion in the body, aggravating the occurrence of infection (30).

The results of the incidence rate of complications at each treatment stage of patients with end-stage DKD under different dialysis methods showed that compared 
with $\mathrm{HD}, \mathrm{PD}$ treatment significantly increased the probability of malignant tumor ( $\mathrm{OR}=1.86,95 \% \mathrm{CI}$ : 1.64 to $2.10 ; \mathrm{P}<0.00001$ ) and significantly increased the risk of complications from the overall probability of complications in patients included in the studies. In the study by Wong et al. (2016) (31), PD was found to increase the incidence of cardiovascular complications, loss of control of blood glucose, lipid metabolism disorders, volume overload, and a series of other complications, seriously affecting the quality of life of patients. It was reported that a series of complications caused by PD treatment are due to insulin resistance, metabolic acidosis, loss of protein from peritoneal dialysate, inflammatory state, and delayed gastric emptying related to autonomic dysfunction (32-35).

It was found from the funnel plots of the meta-analysis on the efficacy and safety of PD for patients with endstage DKD that the publications selected for this study were free of bias, the conclusions obtained were reliable, and the risk of bias was not the main factor affecting the conclusions.

\section{Conclusions}

Four relevant articles on PD for the clinical treatment of patients with end-stage DKD were included in this study. A meta-analysis was performed to confirm that PD could significantly increase the serum creatinine and blood urea nitrogen levels, significantly decrease the serum protein and albumin levels, but significantly increase the probability of complications.

The limitation of this study is that the type of control group in the studies was limited, the sample size of the included studies was small, the result error was large. In addition, the source range of the research objects included in the literature is relatively small, which leads to information bias in the data collection process. By changing the inclusion criteria and excluding low-quality studies, the heterogeneity could not be significantly reduced, which may have a certain impact on the results. In the future, it is necessary to conduct multi-center, large-sample, and high-quality studies on PD treatment for end-stage DKD. In conclusion, this study provides a scientific basis for the efficacy and safety evaluation of PD treatment in patients with end-stage DKD.

\section{Acknowledgments}

Funding: None.

\section{Footnote}

Reporting Checklist: The authors have completed the PRISMA reporting checklist. Available at https://apm. amegroups.com/article/view/10.21037/apm-22-50/rc

Conflicts of Interest: All authors have completed the ICMJE uniform disclosure form (available at https://apm. amegroups.com/article/view/10.21037/apm-22-50/coif). The authors have no conflicts of interest to declare.

Ethical Statement: The authors are accountable for all aspects of the work in ensuring that questions related to the accuracy or integrity of any part of the work are appropriately investigated and resolved.

Open Access Statement: This is an Open Access article distributed in accordance with the Creative Commons Attribution-NonCommercial-NoDerivs 4.0 International License (CC BY-NC-ND 4.0), which permits the noncommercial replication and distribution of the article with the strict proviso that no changes or edits are made and the original work is properly cited (including links to both the formal publication through the relevant DOI and the license). See: https://creativecommons.org/ licenses/by-nc-nd/4.0/.

\section{References}

1. Chow KM, Wong SSM, Ng JKC, et al. Straight Versus Coiled Peritoneal Dialysis Catheters: A Randomized Controlled Trial. Am J Kidney Dis 2020;75:39-44.

2. Li Z, Tang XH, Li Q, et al. Ultrasound-Guided Oblique Sub-Costal Transversus Abdominis Plane Block as the Principal Anesthesia Technique in Peritoneal Dialysis Catheter Implantation and Plasma Ropivacaine Concentration Evaluation in ESRD Patients: A Prospective, Randomized, Double-Blinded, Controlled Trial. Perit Dial Int 2018;38:192-9.

3. Lichodziejewska-Niemierko $M$, Chmielewski M, Dudziak $M$, et al. Hydration Status of Patients Dialyzed with Biocompatible Peritoneal Dialysis Fluids. Perit Dial Int 2016;36:257-61.

4. Tsuruya K, Shimazaki R, Fukagawa M, et al. Efficacy and safety of evocalcet in Japanese peritoneal dialysis patients. Clin Exp Nephrol 2019;23:739-48.

5. Farhat K, Stavenuiter AWD, Vervloet MG, et al. Effects of Oral Paricalcitol and Calcitriol Treatment on Peritoneal 
Membrane Characteristics of Peritoneal Dialysis Patients A Pilot Study. Perit Dial Int 2018;38:220-8.

6. Liu XY, Gao XM, Zhang N, et al. Oral Bicarbonate Slows Decline of Residual Renal Function in Peritoneal Dialysis Patients. Kidney Blood Press Res 2017;42:565-74.

7. Chang CN, Niu CY, Tan AC, et al. The Effect of FarInfrared Therapy on the Peritoneal Expression of Glucose Degradation Products in Diabetic Patients on Peritoneal Dialysis. Int J Mol Sci 2021;22:3732.

8. Borgia SM, Dearden J, Yoshida EM, et al. Sofosbuvir/ velpatasvir for 12 weeks in hepatitis $\mathrm{C}$ virus-infected patients with end-stage renal disease undergoing dialysis. $\mathrm{J}$ Hepatol 2019;71:660-5.

9. Bitencourt Dias D, Mendes ML, Burgugi Banin V, et al. Urgent-Start Peritoneal Dialysis: The First Year of Brazilian Experience. Blood Purif 2017;44:283-7.

10. Chuasuwan A, Pooripussarakul S, Thakkinstian A, et al. Comparisons of quality of life between patients underwent peritoneal dialysis and hemodialysis: a systematic review and meta-analysis. Health Qual Life Outcomes 2020;18:191.

11. Mehrotra R, Devuyst O, Davies SJ, et al. The Current State of Peritoneal Dialysis. J Am Soc Nephrol 2016;27:3238-52.

12. Perl J, Bargman JM. Peritoneal dialysis: from bench to bedside and bedside to bench. Am J Physiol Renal Physiol 2016;311:F999-F1004.

13. Chang TI, Ryu DR, Yoo TH, et al. Effect of Icodextrin Solution on the Preservation of Residual Renal Function in Peritoneal Dialysis Patients: A Randomized Controlled Study. Medicine (Baltimore) 2016;95:e2991.

14. Baek HS, Park KS, Ha IS, et al. Impact of end-stage renal disease in children on their parents. Nephrology (Carlton) 2018;23:764-70.

15. De Los Ríos T, Pérez-Martínez J, Portoles J, et al. Effect of balance Solution on the Peritoneal Membrane in Automated Peritoneal Dialysis. Perit Dial Int 2016;36:569-72.

16. Singh T, Astor BC, Waheed S. End-Stage Renal Disease Patients with Low Serum Albumin: Is Peritoneal Dialysis an Option? Perit Dial Int 2019;39:562-7.

17. Wong B, Ravani P, Oliver MJ, et al. Comparison of Patient Survival Between Hemodialysis and Peritoneal Dialysis Among Patients Eligible for Both Modalities. Am J Kidney Dis 2018;71:344-51.

18. Choi JY, Jang HM, Park J, et al. Survival advantage of peritoneal dialysis relative to hemodialysis in the early period of incident dialysis patients: a nationwide prospective propensity-matched study in Korea. PLoS One 2013;8:e84257.

19. Lee JH, Park SH, Lim JH, et al. Impact of dialysis modality on technique survival in end-stage renal disease patients. Korean J Intern Med 2016;31:106-15.

20. Jin H, Ni Z, Che X, et al. Peritoneal Dialysis as an Option for Unplanned Dialysis Initiation in Patients with EndStage Renal Disease and Diabetes Mellitus. Blood Purif 2019;47:52-7.

21. Wang IK, Shen TC, Muo CH, et al. Risk of pulmonary embolism in patients with end-stage renal disease receiving long-term dialysis. Nephrol Dial Transplant 2017;32:1386-93.

22. Ekart R, Hojs R. Obese and diabetic patients with endstage renal disease: Peritoneal dialysis or hemodialysis? Eur J Intern Med 2016;32:1-6.

23. Htay H, Cho Y, Pascoe EM, et al. Predictors of Residual Renal Function Decline in Peritoneal Dialysis Patients: The balANZ Trial. Perit Dial Int 2017;37:283-9.

24. Nataatmadja M, Cho Y, Pascoe EM, et al. Association Between Peritoneal Glucose Exposure and Peritonitis in Peritoneal Dialysis Patients: The balANZ Trial. Perit Dial Int 2017;37:407-13.

25. Ye H, Yang X, Yi C, et al. Urgent-start peritoneal dialysis for patients with end stage renal disease: a 10-year retrospective study. BMC Nephrol 2019;20:238.

26. Ranganathan D, John GT, Yeoh E, et al. A Randomized Controlled Trial to Determine the Appropriate Time to Initiate Peritoneal Dialysis after Insertion of Catheter (Timely PD Study). Perit Dial Int 2017;37:420-8.

27. Xue J, Li H, Zhou Q, et al. Comparison of peritoneal dialysis with hemodialysis on survival of diabetic patients with end-stage kidney disease: a meta-analysis of cohort studies. Ren Fail 2019;41:521-31.

28. Rago C, Lombardi T, Di Fulvio G, et al. A New Peritoneal Dialysis Solution Containing L-Carnitine and Xylitol for Patients on Continuous Ambulatory Peritoneal Dialysis: First Clinical Experience. Toxins (Basel) 2021;13:174.

29. Sikaneta T, Wu G, Abdolell M, et al. The Trio Trial A Randomized Controlled Clinical Trial Evaluating the Effect of a Biocompatible Peritoneal Dialysis Solution on Residual Renal Function. Perit Dial Int 2016;36:526-32.

30. Javaid MM, Khan BA, Subramanian S. Peritoneal dialysis as initial dialysis modality: a viable option for late-presenting end-stage renal disease. J Nephrol 2019;32:51-6.

31. Wong PN, Tong GM, Wong YY, et al. Alternating Mupirocin/Gentamicin is Associated with Increased Risk 
of Fungal Peritonitis as Compared with Gentamicin Alone - Results of a Randomized Open-Label Controlled Trial. Perit Dial Int 2016;36:340-6.

32. Akizawa T, Otsuka T, Reusch M, et al. Intermittent Oral Dosing of Roxadustat in Peritoneal Dialysis Chronic Kidney Disease Patients with Anemia: A Randomized, Phase 3, Multicenter, Open-Label Study. Ther Apher Dial 2020;24:115-25.

33. Yan H, Fang W, Lin A, et al. Three Versus 4 Daily Exchanges and Residual Kidney Function Decline in Incident CAPD Patients: A Randomized Controlled Trial. Am J Kidney Dis 2017;69:506-13.

Cite this article as: $\mathrm{Wu} \mathrm{H}$, Zhou H, Zhang Q, Zhou Y, Fu L, Zhuang Y. Systematic review and meta-analysis: the effect and safety of peritoneal dialysis in patients with end-stage diabetic kidney disease. Ann Palliat Med 2022;11(2):695-707. doi: 10.21037/apm-22-50
34. Kitrungphaiboon T, Puapatanakul P, Chuengsaman P, et al. Intraperitoneal Cefepime Monotherapy Versus Combination Therapy of Cefazolin Plus Ceftazidime for Empirical Treatment of CAPD-Associated Peritonitis: A Multicenter, Open-Label, Noninferiority, Randomized, Controlled Trial. Am J Kidney Dis 2019;74:601-9.

35. Vural A, Koçyiğit İ, Şan F, et al. Long-Term Protective Effect of N-Acetylcysteine against Amikacin-Induced Ototoxicity in End-Stage Renal Disease: A Randomized Trial. Perit Dial Int 2018;38:57-62.

(English Language Editor: C. Betlazar-Maseh) 